\title{
Observations and modeling of GIC in the Chinese large-scale high-voltage power networks
}

\author{
Chunming $\mathrm{Liu}^{1,2}$, Yunlong $\mathrm{Li}^{1}$, and Risto Pirjola ${ }^{2,3}$ \\ 1 School of Electrical and Electronic Engineering, North China Electric Power University, Beijing, China \\ *Corresponding author: LiuChunming@ncepu.edu.cn; Chunming.Liu@NRCan-RNCan.gc.ca \\ 2 Geomagnetic Laboratory, Natural Resources Canada, Ottawa, Canada \\ 3 Finnish Meteorological Institute, Helsinki, Finland
}

Received 28 March 2013 / Accepted 17 December 2013

\begin{abstract}
During geomagnetic storms, the geomagnetically induced currents (GIC) cause bias fluxes in transformers, resulting in half-cycle saturation. Severely distorted exciting currents, which contain significant amounts of harmonics, threaten the safe operation of equipment and even the whole power system. In this paper, we compare GIC data measured in transformer neutrals and magnetic recordings in China, and show that the GIC amplitudes can be quite large even in mid-low latitude areas. The GIC in the Chinese Northwest $750 \mathrm{kV}$ Power Grid are modeled based on the plane wave assumption. The results show that GIC flowing in some transformers exceed $30 \mathrm{~A} /$ phase during strong geomagnetic storms. GIC are thus not only a high-latitude problem but networks in middle and low latitudes can be impacted as well, which needs careful attention.
\end{abstract}

Key words. electric circuit - geomagnetically induced currents (GIC) - modelling - engineering - space weather

\section{Introduction}

During strong space weather storms, which are caused by the activity of the Sun, the Earth's magnetic field is intensely disturbed by the space current system in the magnetosphere and ionosphere. The electric fields induced by time variations of the geomagnetic field drive geomagnetically induced currents (GIC) in electric power transmission networks. The frequencies of GIC are in the range of $0.0001 \sim 0.1 \mathrm{~Hz}$. Such quasi-DC currents cause bias fluxes in transformers, which result in half-cycle saturation due to the nonlinear response of the core material (e.g., Kappenman \& Albertson 1990; Molinski 2002; Kappenman 2007). The sharply increased magnetizing current with serious waveform distortion may lead to temperature rise and vibration in transformers, reactive power fluctuations, voltage sag, protection relay malfunction, and possibly even a collapse of the whole power system (e.g., Kappenman 1996; Bolduc 2002).

Large GIC are usually considered to occur at high latitudes such as North America and Scandinavia, where tripping problems and even blackouts of power systems due to GIC have been experienced (Bolduc 2002; Pulkkinen et al. 2005; Wik et al. 2009). Large currents in transformer neutrals have been monitored in the Chinese high-voltage power system many times during geomagnetic storms although China is a midlow-latitude country. At the same time, transformers have had abnormal noise and vibration. Those events have been shown to be caused by GIC based on analyses of simultaneous magnetic data and GIC recordings (Liu \& Xie 2005; Liu et al. 2009a). The power grids are using higher voltages, longer transmission distances, and larger capacity with the developing economy in China. So, the risk that the power systems would suffer from GIC problems may obviously increase. The Chinese Northwest $750 \mathrm{kV}$ power grid has long transmission lines with small resistances making it prone to large GIC during geomagnetic storms. Thus it is important to model GIC particularly in that network.

\section{GIC observations in Chinese high-voltage power grid}

We acquire GIC data through the neutral point of the transformer at the Ling'ao nuclear power plant $\left(22.6^{\circ} \mathrm{N}\right.$, $\left.114.6^{\circ} \mathrm{E}\right)$ in the Guangdong Province. Besides, geomagnetic field data are collected from the Zhaoqing Geomagnetic Observatory $\left(23.1^{\circ} \mathrm{N}, 112.3^{\circ} \mathrm{E}\right)$ which is not very far from Ling'ao. Figurel shows the neutral point current (top panel), the horizontal component of the geomagnetic field (bottom panel), and its variation rate (middle panel) during the magnetic storms on 7-8 (a) and 9-10 (b) November 2004. The occurrence times of the current peaks match with those of the geomagnetic field variation rate. It is confirmed that there is no HVDC (high-voltage direct current) monopole operation during that time. So it is reasonable to believe that the currents are really GIC induced by geomagnetic storms. The maximum value of GIC is up to $75.5 \mathrm{~A} / 3$ phases, which is much higher than the DC bias caused by monopole operation of HVDC.

\section{Modeling GIC in power grids}

The modeling of GIC in a power grid can be divided into two steps (e.g., Pirjola 2000): step 1, calculating the geoelectric field induced by a magnetic storm; step 2, calculating the GIC in the power grid. The effect of the induced geoelectric field is equivalent to voltage sources in the transmission lines, which enables converting the GIC calculation into a circuit problem in step 2. 


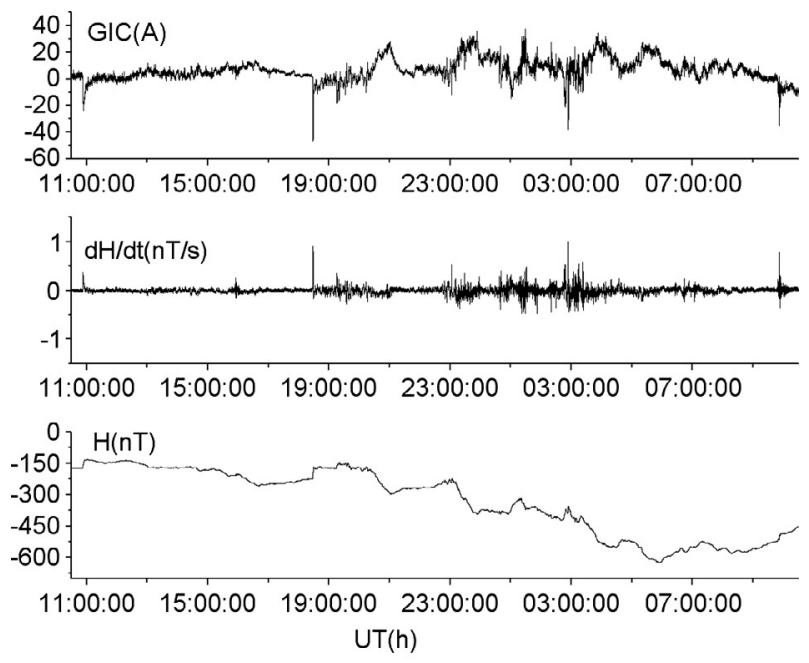

(a)
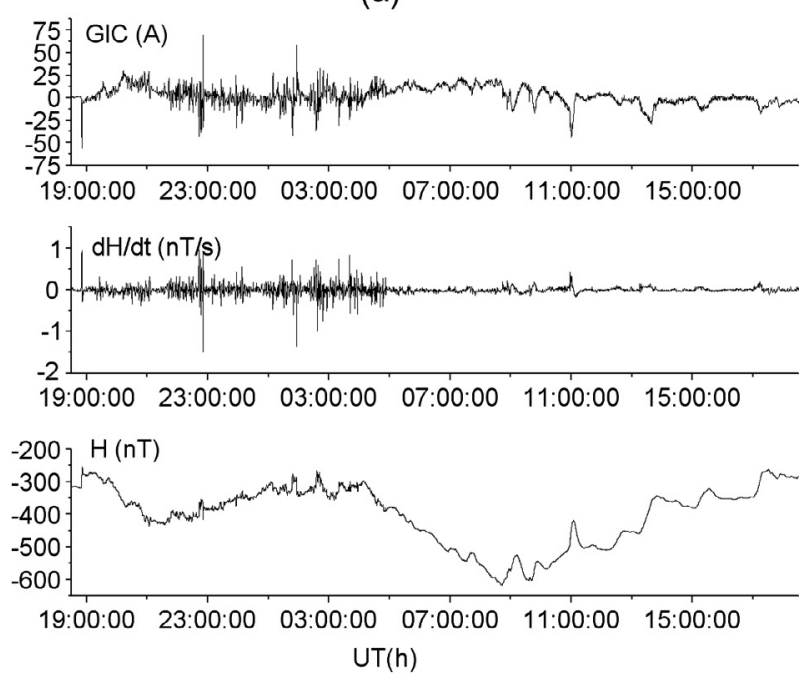

(b)

Fig. 1. GIC data at the Ling'ao nuclear power plant on 7-8 (a) and 9-10 (b) November 2004. The horizontal component of the geomagnetic field and its variation rate are also shown based on data from the Zhaoqing Geomagnetic Observatory.

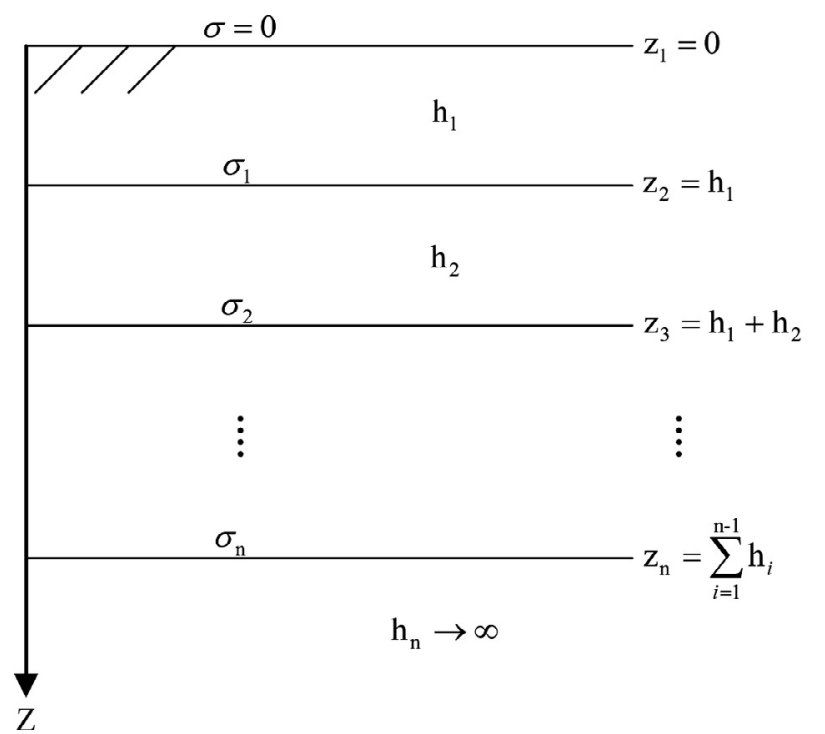

Fig. 2. Layered Earth model for calculating the induced geoelectric field.

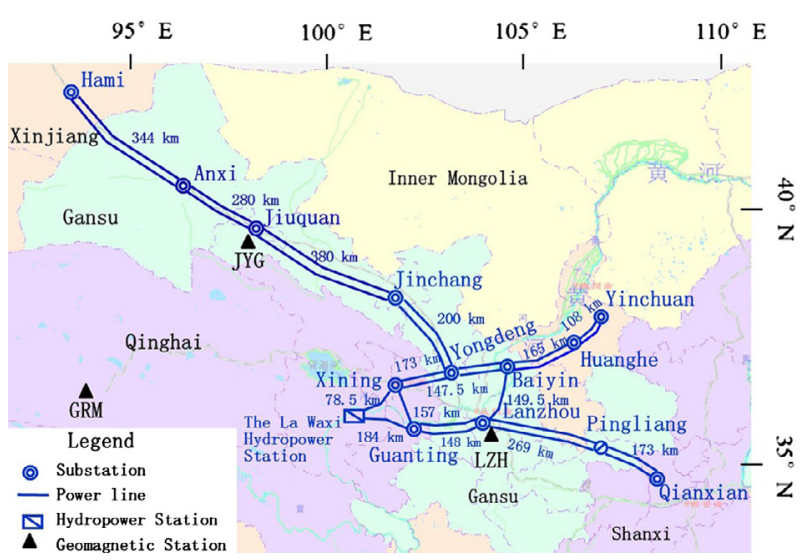

Fig. 3. Chinese Northwest $750 \mathrm{kV}$ power grid. Three geomagnetic observatories (GRM, LZH, and JYG) are also shown on the map. (The WMQ observatory is not located in the area of this map.)

Table 1. Locations of geomagnetic observatories in the area of the Chinese Northwest $750 \mathrm{kV}$ power grid.

\begin{tabular}{lcc}
\hline \hline Name & Longitude $\left({ }^{\circ} \mathrm{E}\right)$ & Latitude $\left({ }^{\circ} \mathrm{N}\right)$ \\
\hline WMQ & 87.7 & 43.8 \\
GRM & 94.9 & 36.4 \\
LZH & 103.8 & 36.1 \\
JYG & 98.2 & 39.8 \\
\hline
\end{tabular}

\subsection{Calculating the electric field using a layered earth model}

We use the standard conventional Cartesian geomagnetic coordinate system in which the $x, y$ and $z$ axes point northwards, eastwards, and downwards, respectively. According to the plane wave assumption (e.g., Boteler 1999), the relation between perpendicular horizontal components of the geoelectric $(E)$ and geomagnetic $(B)$ fields at the earth's surface can be expressed as

$$
\begin{gathered}
E_{x}(\omega)=\frac{1}{\mu_{0}} B_{y}(\omega) Z(\omega), \\
E_{y}(\omega)=-\frac{1}{\mu_{0}} B_{x}(\omega) Z(\omega),
\end{gathered}
$$

where $\mu_{0}$ is the vacuum permeability and $Z$ is surface impedance of the earth which depends on the conductivity structure of the earth and on the angular frequency $\omega$.

In a previous study about GIC in China, Liu et al. (2009b) used a uniform half-space model for the earth. However, one-dimensional layered earth models are more accurate descriptions for the real situations. Figure 2 shows a layered earth model which contains $n$ layers with conductivities $\sigma_{1}$, $\sigma_{2}, \ldots, \sigma_{n}$ and thicknesses $h_{1}, h_{2}, \ldots, h_{n} \rightarrow \infty$.

The thickness of the bottom layer is $h_{n} \rightarrow \infty$, and $E_{x}=0$ and $B_{y}=0$ when $z \rightarrow \infty$. Hence the impedance at the top of the layer of the $n$th layer is

$$
Z_{n}=\mu_{0} \frac{E_{x}}{B_{y}}=\frac{j \omega \mu_{0}}{k_{n}}=\sqrt{\frac{j \omega \mu_{0}}{\sigma_{n}}},
$$

where $k_{n}$ is the propagation constant given by $k_{n}=\sqrt{j \omega \mu_{0} \sigma_{n}}$. The impedance at the top of the layer within the $m$ th layer $(m=1,2, \ldots, n-1)$ can be expressed as 
C. Liu et al.: Observations and modeling of GIC in Chinese power networks
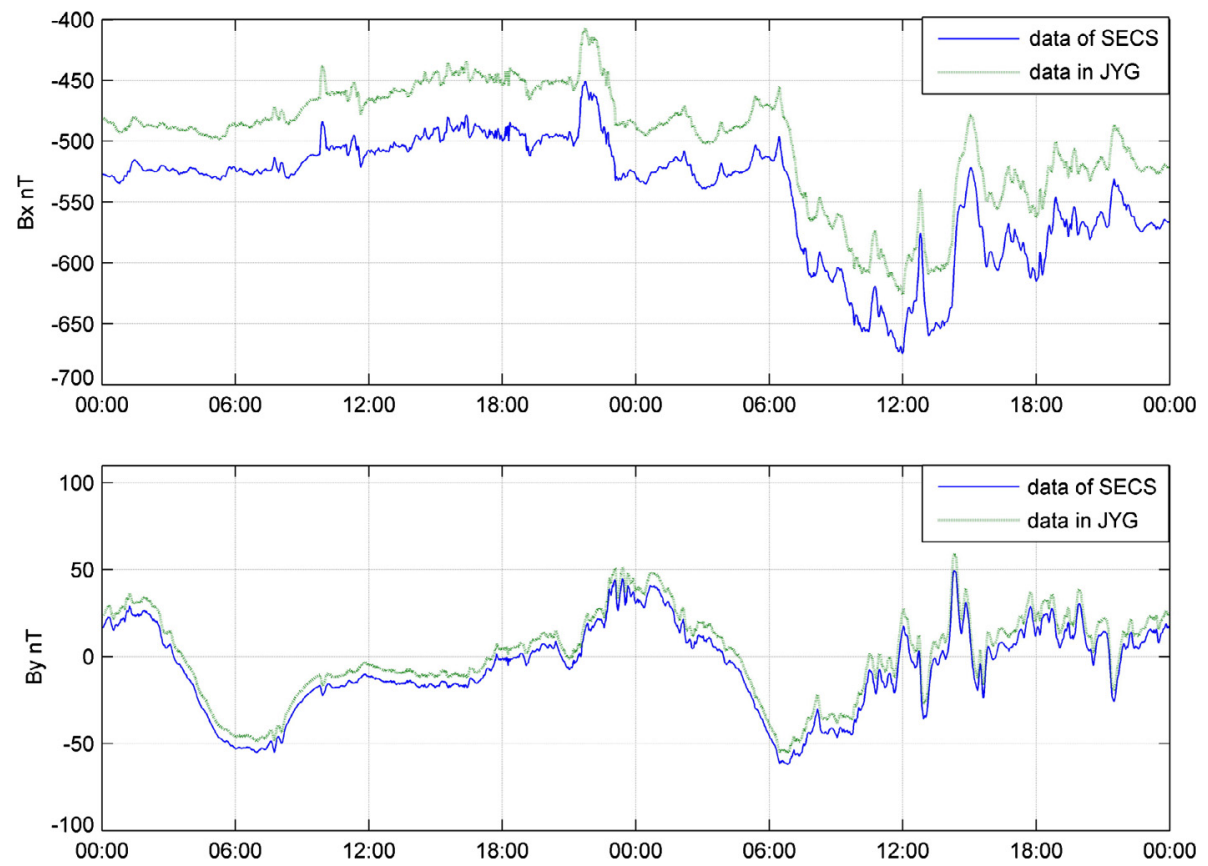

(a)
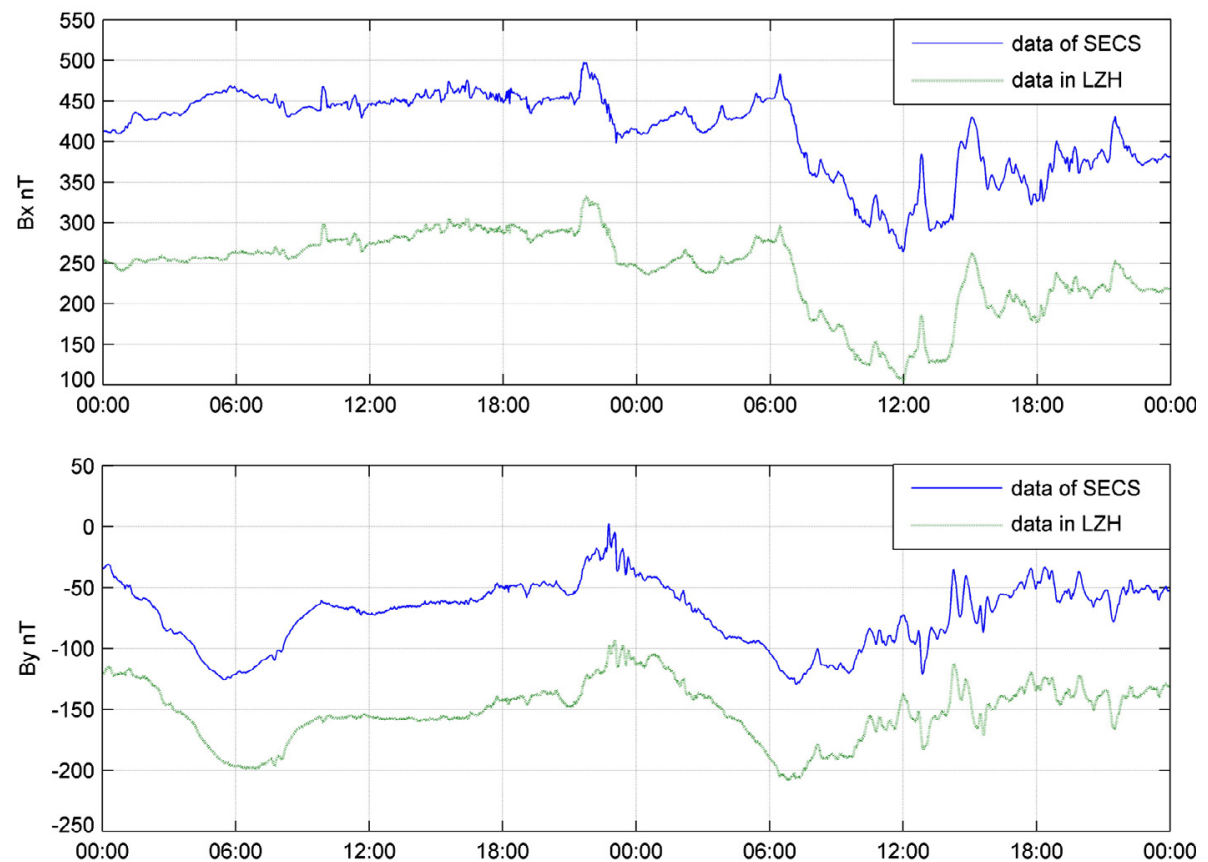

(b)

Fig. 4. Measured magnetic data and the SECS-derived magnetic data on 29-30 May 2005. The horizontal axis is the UT time in hours (a) magnetic data from JYG observatory and the SECS-derived magnetic data for Jiuquan substation and (b) magnetic data from LZH observatory and the SECS-derived magnetic data for Yongdeng substation.

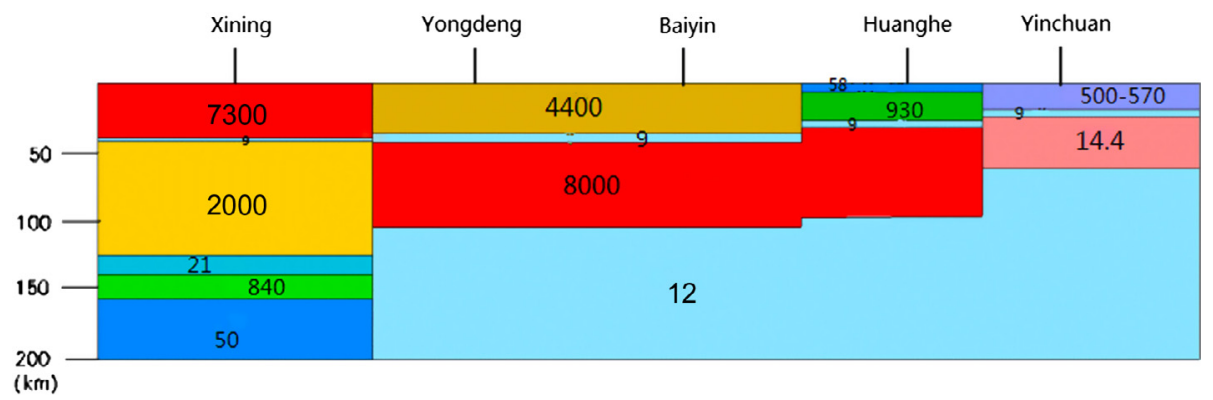

Fig. 5. Resistivity for the section Xining-Yinchuan along $750 \mathrm{kV}$ power transmission lines. 

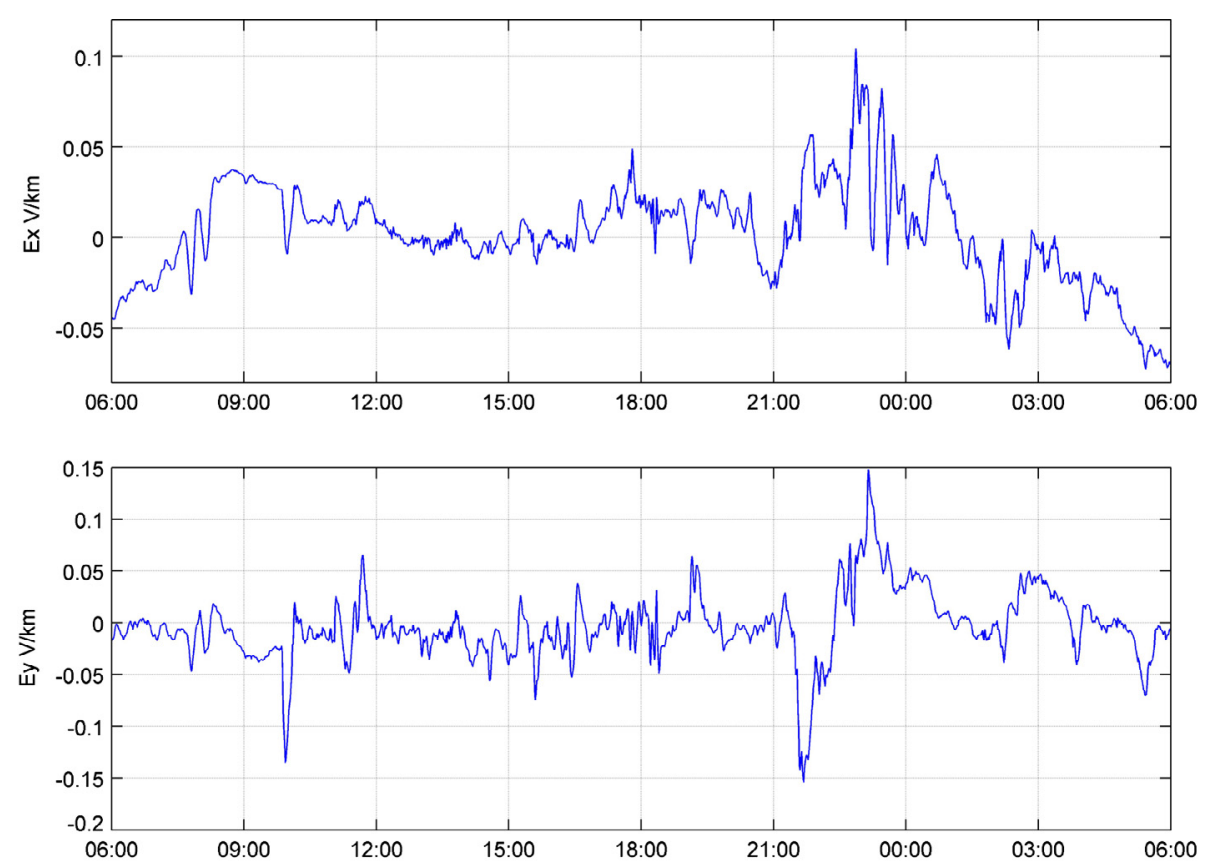

(a)
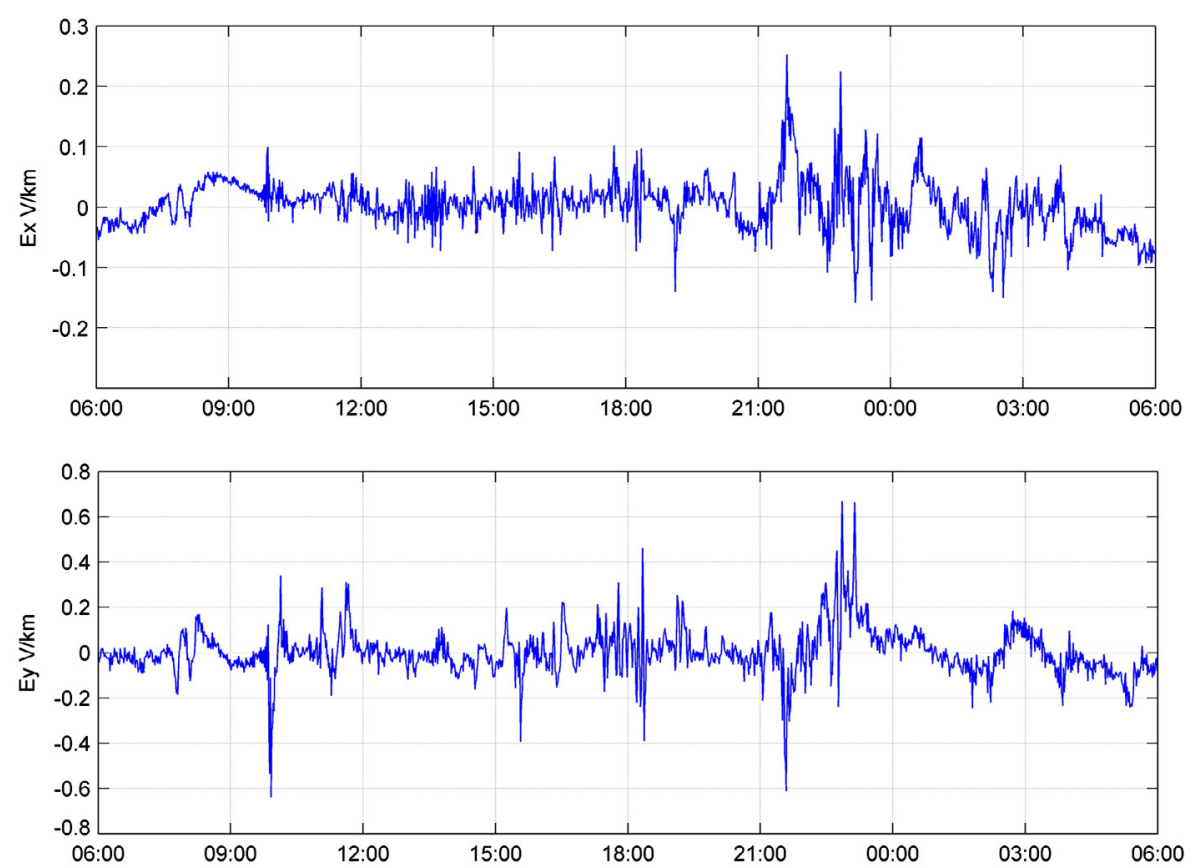

(b)

Fig. 6. Calculated geoelectric fields at two sites (Jiuquan and Yongdeng) of the Chinese Northwest $750 \mathrm{kV}$ power grid on 29-30 May 2005. The horizontal axis is the UT time in hours (a) E-Jiuquan and (b) E-Yongdeng.

$$
Z_{m}=Z_{0 m} \frac{1-L_{m+1} e^{-2 k_{m} h_{m}}}{1+L_{m+1} e^{-2 k_{m} h_{m}}}
$$

where $k_{m}=\sqrt{j \omega \mu_{0} \sigma_{m}}$ and $Z_{0 m}=\frac{j w \mu_{0}}{k_{m}}$ and $L_{m+1}=\frac{Z_{0 m}-Z_{m+1}}{Z_{0 m}+Z_{m+1}}$.

In the model, the bottom of $m$ th layer is the top of $(m+1)$ th layer, so equation (4) can be seen as a recursive formula for the impedance at the top of each layer, through which we can calculate the surface impedance of the Earth $Z$. The geoelectric field in frequency domain can be calculated from geomagnetic data according to equations (1) and (2). Then the result has to be inverse Fourier transformed back to the time domain.

\subsection{Calculating GIC}

The frequencies of GIC are very low from the view point of power systems. Thus the GIC can be treated as a direct current. The effect of the geoelectric field on a power grid is equivalent to a set of voltage sources in the transmission lines between the substations. The value of the voltage is the integral of the electric field along the line, i.e.:

$$
V_{A B}=\int_{A}^{B} \vec{E} \cdot \overleftarrow{\mathrm{d} l} .
$$




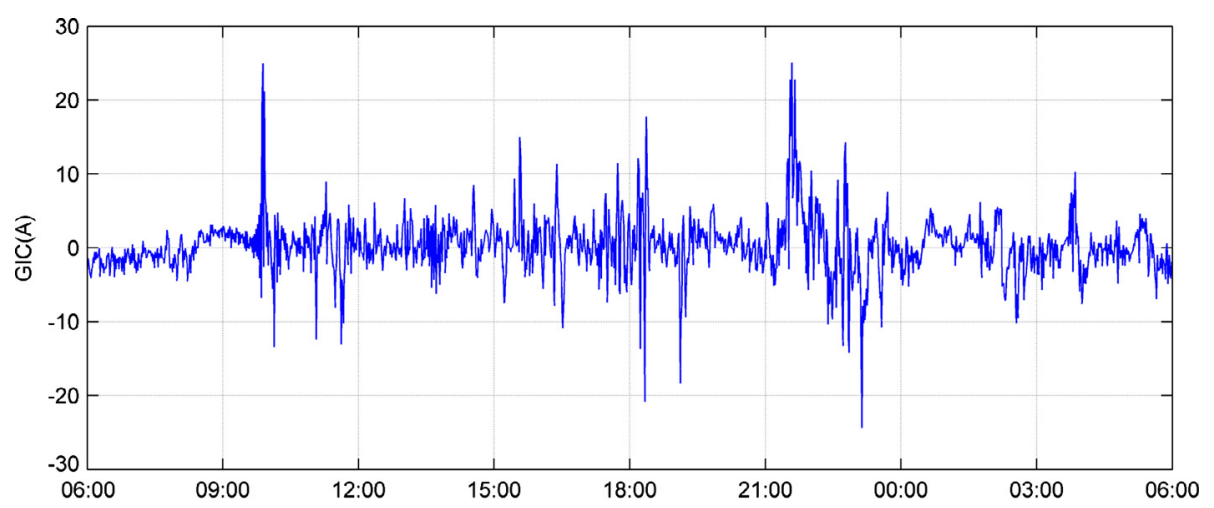

(a)

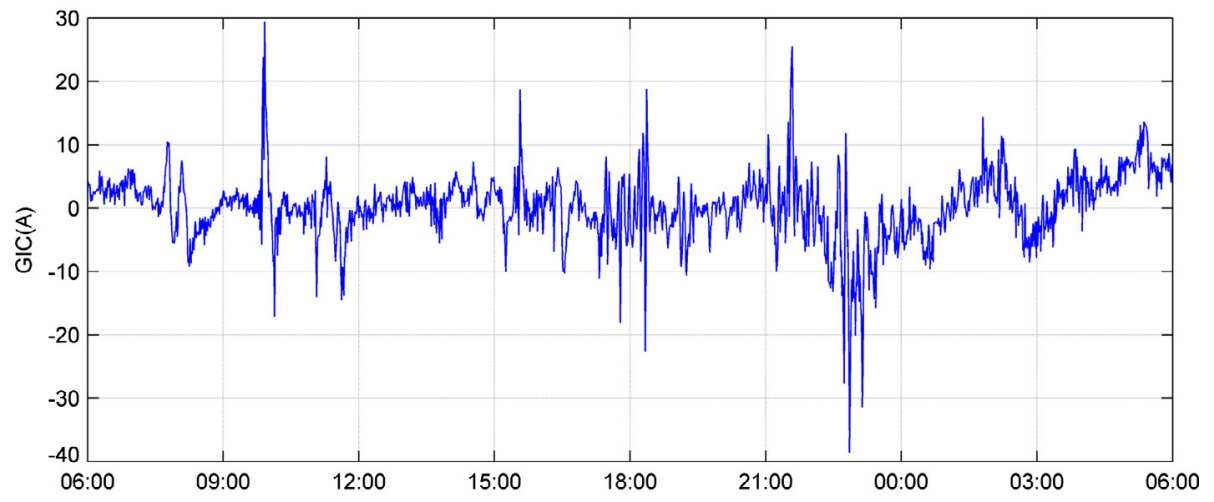

(b)

Fig. 7. Calculated GIC at two sites (Jiuquan and Yongdeng) of the Chinese Northwest $750 \mathrm{kV}$ power grid on 29-30 May 2005. The horizontal axis is the UT time in hours (a) calculated GIC at Jiuquan substation and (b) calculated GIC at Youngdeng substation.

If the geoelectric field is uniform, the integrals are independent of the paths. Therefore equation (5) can be simplified to

$$
V_{A B}=L_{A B}\left(E_{x} \sin \theta+E_{y} \cos \theta\right)
$$

Where $L_{A B}$ is the direct distance between nodes $A$ and $B ; \theta$ is the "compass angles" i.e. clockwise from geographic North.

The GIC flowing from the power grid to the earth can be expressed as a column matrix I, which has the following formula (e.g., Pirjola \& Lehtinen 1985)

$$
\mathbf{I}=(\mathbf{1}+\mathbf{Y Z Z})^{-1} \mathbf{J}
$$

where $\mathbf{1}$ is a unit (identity) matrix; $\mathbf{Y}$ and $\mathbf{Z}$ are the network admittance matrix and the earthing impedance matrix respectively. The elements of column matrix $\mathbf{J}$ are defined by

$$
J_{i}=\sum_{j=1, j \neq i}^{N} \frac{V_{i j}}{R_{i j}} .
$$

The matrix $\mathbf{J}$ gives the GIC between the power grid and the earth in the case of ideal groundings, i.e. the grounding resistances are zero making $\mathbf{Z}$ a zero matrix.

\section{Modeling GIC in Chinese Northwest 750 kV power grid}

The problem of GIC should be considered more serious in the Chinese Northwest $750 \mathrm{kV}$ power grid because of the high voltage implying low transmission line resistances and because of the low earth conductivity increasing geoelectric field values. The power grid (shown in Fig. 3) for which GIC calculations are made in this paper is mainly located in the Gansu Province in the Northwest of China. We ignore the lower voltage part connected to the $750 \mathrm{kV}$ power grid when modeling the GIC, because the resistances of that part are much larger, and so it is considered to have little influence on GIC flowing in the $750 \mathrm{kV}$ system.

\subsection{Geoelectric field calculation}

We use data of the geomagnetic storm on 29-30 May 2005. The power grid is very large, extending more than $2000 \mathrm{~km}$ in an east-west direction and $1500 \mathrm{~km}$ in a North-South direction, so the geomagnetic variations cannot be considered to be the same all over the network. The magnetic data from four geomagnetic observatories, whose locations are shown in Figure 3 and in Table 1, are used to calculate the geoelectric field. The local magnetic data are interpolated by using the spherical elementary current systems (SECS) method (Amm 1997). The method uses geomagnetic field data to inverse the ionosphere equivalent current according to which the geomagnetic field data of every location can be calculated. Therefore the interpolation of magnetic data at different locations during a storm can be acquired. As examples, Figure 4a shows the measured data from JYG and the SECS-derived magnetic data for Jiuquan Substation, and Figure $4 \mathrm{~b}$ shows the measured data from LZH and the SECS-derived magnetic data for Yongdeng Substation on 29-30 May 2005. It can be seen that the differences between measured magnetic data and the SECS-derived 


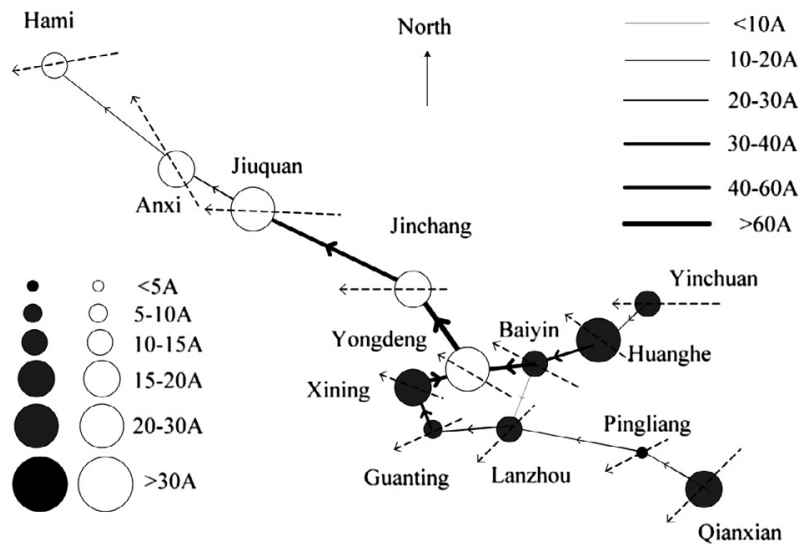

(a) Calculated GIC results at 21:35UT 29 May 2005

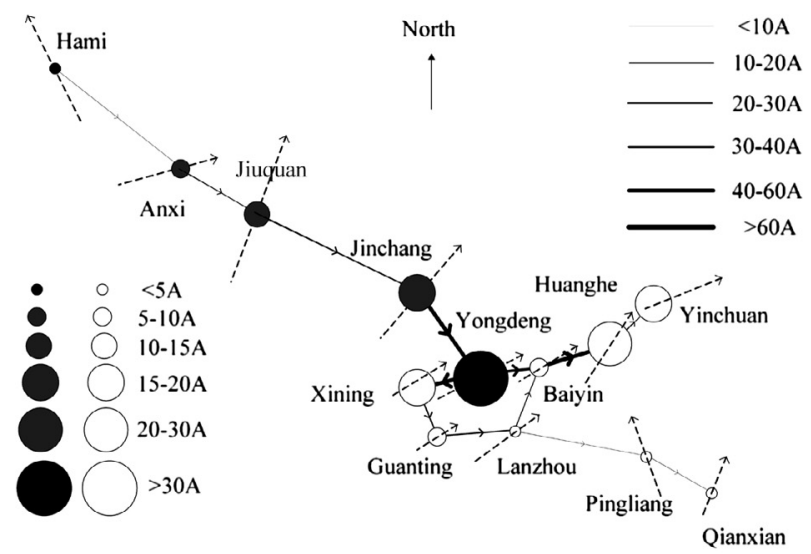

(b) Calculated GIC results at 22:51UT 29 May 2005

Fig. 8. Snapshots at $21: 35$ on 29 May 2005 (a) and at 22:51UT on 29 May 2005 (b) of calculated GIC at different sites of the Chinese Northwest $750 \mathrm{kV}$ power grid. The solid circle represents that the GIC flow into the power network from ground, the hollow one means that GIC flow into the ground. The dashed line with an arrow represents the direction of electric field at that substation.

data are little except for the base line values which have no effect on the induced electric fields.

The earth conductivities are quite different across the power grid considered, so the geoelectric field values are calculated segment by segment according to the local magnetic data and the local layered earth model. In other words, we utilize the piecewise layered earth model. The earth resistivity in the region where the Chinese Northwest $750 \mathrm{kV}$ power grid is located was provided by Prof. Liu Guo-Xing, a geologist at the Jilin University (private communication). Figure 5 shows a section of the earth resistivity in $\Omega \cdot \mathrm{m}$ from Xining to Yinchuan along the $750 \mathrm{kV}$ power lines (see Fig. 3). The resistances of some places are given within a range such as 500-570 at Yinchuan in Figure 5. The upper limit values were used to calculate the induced electric fields because they stand for the most disadvantageous situation to the power grid.

As mentioned, the geoelectric fields have been calculated all over the Chinese Northwest $750 \mathrm{kV}$ system based on the Piecewise layered earth models during the geomagnetic storm on 29-30 May 2005. As examples, Figure 6 shows the geoelectric field at Jiuquan and Yongdeng (whose locations are shown in Fig. 3). Our calculation results indicate that the largest $E_{x}$ value is $0.36 \mathrm{~V} / \mathrm{km}$ and the largest $E_{y}$ value is $0.668 \mathrm{~V} / \mathrm{km}$ in the area of the Northwest $750 \mathrm{kV}$ grid during the geomagnetic storm considered. It is also shown by Figure 6 that the electric fields calculated for Yongdeng and Jiugan are quite different because the Earth conductivity at Yongdeng is much lower than that at Jiuquan.

\subsection{GIC calculation}

The GIC through all neutral points of the transformers to the Earth and in all transmission lines of the Chinese Northwest $750 \mathrm{kV}$ network have been calculated. Figure 7 shows the GIC through two typical substations: Jiuquan and Yondeng (also referred to in Fig. 6). The largest GIC at Jiuquan is $25.08 \mathrm{~A} /$ phase at 21:35 UT on 29 May 2005, and the largest GIC at Yongdeng is $38.63 \mathrm{~A} /$ phase at 22:51 UT on 29 May 2005.

As snapshots, Figure 8 shows the GIC through every node and line at 21:35 UT (panel a) and at 22:51UT (panel b) on 29 May 2005 when the GIC through some of the nodes reach their peaks. It can be seen that the largest GIC through a neutral point is $38.63 \mathrm{~A} /$ phase, which is obtained at theYongdeng substation at 22:51 as already mentioned above (see also Fig. 7). The peak GIC through a transmission line is $68.84 \mathrm{~A} /$ phase, which occurs in the line from Yongdeng to Jinchang at 21:35 UT. It should be note that there is one single-phase transformer bank in a $750 \mathrm{kV}$ substation except Guanting and Yinchuan where the number of transformer banks is two.

\section{Conclusions}

The high-voltage power grid in China may experience large GIC during geomagnetic storms, which has been concluded from monitoring the current through the neutral point at Ling'ao nuclear power plant. The GIC in the Chinese Northwest $750 \mathrm{kV}$ power grid during a specific geomagnetic storm have been modeled based on calculating the geoelectric field using the piecewise layered earth models. It can be seen from the results that some sites are sensitive to geomagnetic storms, and the magnitude of GIC can be quite large (> $30 \mathrm{~A} /$ phase) during strong geomagnetic storms. Our studies thus clearly demonstrate that GIC are not only a high-latitude problem but networks in middle and low latitudes can be impacted as well. Factors increasing GIC risks in China include the large size of the power network, the small resistances of the transmission lines, and the high resistivity of the earth.

Acknowledgements. This work was supported by the Nature Science Foundation of Hebei Province (E2011502085), National High Technology Research and Development of China 863 Program (2012AA121005).

\section{References}

Amm, O., Ionospheric elementary current systems in spherical coordinates and their application, J. Geomag. Geoelectr., 49, 947-955, 1997.

Bolduc, L., GIC observations and studies in the Hydro-Québec power system, J. Atmos. Sol. Terr. Phys., 64 (16), 1793-1802, 2002.

Boteler, D.H., Calculating the voltages induced in technological systems during a geomagnetic disturbance, IEEE Trans. Electromagn. Compat., 41 (4), 398-402, 1999.

Kappenman, J.G., Geomagnetic storms and their impact on power systems, IEEE Power Eng. Rev., 16 (5), 5-8, 1996. 


\section{Liu et al.: Observations and modeling of GIC in Chinese power networks}

Kappenman, J.G., Geomagnetic disturbances and impacts upon power system operation. In: The Electric Power Engineering Handbook, L.L., Grigsby (Ed.), 2nd Edn., Chapter 16, 16-1 16-22, CRC Press/IEEE Press, 2007.

Kappenman, J.G., and V.D. Albertson, Bracing for the geomagnetic storms, IEEE Spectrum, The Electric Power Engineering Handbook, 27(3), 27-33, 1990.

Liu, L.Y., and X.W. Xie, Analysis of increase of noise of $500 \mathrm{kV}$ transformer (in Chinese), High Voltage Engineering, 31 (4), $85-87,2005$.

Liu, C.-M., L.-G. Liu, and R. Pirjola, Geomagnetically induced currents in the high-voltage power grid in China, IEEE Trans. Power Delivery, 24 (4), 2368-2374, 2009a.

Liu, C.-M., L.-G. Liu, R. Pirjola, and Z.-Z. Wang, Calculation of geomagnetically induced currents in mid- to low-latitude power grids based on the plane wave method: A preliminary case study, Space Weather, 7 (4), S04005, DOI: 10.1029/2008SW000439, 9 pp., 2009b.
Molinski, T.S., Why utilities respect geomagnetically induced currents, J. Atmos. Sol. Terr. Phys., 64 (16), 1765-1778, 2002.

Pirjola, R., Geomagnetically Induced Currents during Magnetic Storms, IEEE Trans. Plasma Sci., 28 (6), 1867-1873, 2000.

Pirjola, R., and M. Lehtinen, Currents produced in the Finnish 400 $\mathrm{kV}$ power transmission grid and in the Finnish natural gas pipeline by geomagnetically induced electric fields, Ann. Geophys., 3 (4), 485-491, 1985.

Pulkkinen, A., S. Lindahl, A. Viljanen, and R. Pirjola, Geomagnetic storm of 29-31 October 2003: Geomagnetically induced currents and their relation to problems in the Swedish high-voltage power transmission system, Space Weather, 3 (8), S08C03, DOI: 10.1029/2004SW000123, 19 pp., 2005.

Wik, M., R. Pirjola, H. Lundstedt, A. Viljanen, P. Wintoft, and A. Pulkkinen, Space weather events in July 1982 and October 2003 and the effects of geomagnetically induced currents on Swedish technical systems, Ann. Geophys., 27 (4), 1775-1787, 2009.

Cite this article as: Liu C, Li Y \& Pirjola R: Observations and modeling of GIC in the Chinese large-scale high-voltage power networks. J. Space Weather Space Clim., 2014, 4, A03. 